\title{
Optimizing hematopoietic stem cell engraftment: a novel role for thrombopoietin
}

\author{
Catherine M. Verfaillie \\ Stem Cell Institute, Division of Hematology, Oncology and Transplantation, Department of Medicine, University of Minnesota \\ Medical School, Minneapolis, Minnesota, USA
}

Address correspondence to: Catherine M. Verfaillie, University of Minnesota, MMC 716, 420 Delaware Street SE, Minneapolis, Minnesota 55455, USA. Phone: (612) 625-0602; Fax: (612) 624-2436; E-mail: verfa001@umn.edu.

J. Clin. Invest. 110:303-304 (2002). doi:10.1172/JCI200216376.

Hematopoietic stem cells (HSC) and progenitors are commonly used to replace the hematopoietic system in patients with hematopoietic malignancies, or patients undergoing high dose chemotherapy. Committed progenitors are responsible for the initial hematopoietic recovery, whereas the long-term repopulating HSC is responsible for establishing life-long multilineage hematopoiesis (1). Although hematopoietic cell transplantations have been used for several decades, it is still not fully understood what governs the different steps required for reestablishing hematopoiesis. HSCs must gain access to the bone marrow (BM) in a process termed homing, take up residence in the $\mathrm{BM}$, undergo selfrenewing cell divisions to produce a larger pool of HSCs, and differentiate to generate more committed progenitors (Figure 1). This is shown most dramatically when single mouse HSCs are transplanted in lethally irradiated recipients. Multilineage hematopoiesis can be reconstituted following transplantation of a single HSC, and BM from these primary recipients can be transferred to multiple secondary recipients, demonstrating the impressive ability of the BM microenvironment to induce HSC expansion as well as differentiation (2). Poor engraftment can result from failure of HSCs to home to the $\mathrm{BM}$, to survive within the BM, or to proliferate and differentiate in the BM.

Under steady state conditions, HSCs reside in the $\mathrm{BM}$ among other hematopoietic cells and non-hematopoietic, mesenchymal cells present in the BM, as well as with more transient neighbors, such as lymphocytes. The concerted action of cell-cell interactions and soluble factors secreted locally or at a distance regulate HSCs and their progeny. However, the exact signals that govern in particular the self-renewal of HSCs are still not fully understood.

Despite numerous studies testing the effect of cytokines on HSCs in vitro and some insights from mouse genetics, little is known of HSC selfrenewal factors. Several cytokines have now been identified that, at least in the whole animal, contribute to HSC expansion, self-renewal, and maintenance. The first is the c-Kit-ligand (KL), also termed stem cell factor (SCF) (3). Most HSCs express c-Kit, and in vitro KL affects proliferation and differentiation of primitive progenitors. Spontaneous mutations of both c-Kit (Steel locus, or $S l$ ) and KL (white spotted locus, or $W$ ) exist in mice. Homozygous loss of either $\mathrm{Sl}$ or $W$ is embryonic lethal, although some alleles of $S l$, such as $S l^{\mathrm{d}}$, or of $W$ (see refs. 3 and 4) cause significant defects in erythropoiesis and mast cell generation but are compatible with life. In both mutant animals, careful enumeration of the HSC pool demonstrates that the number of long-term repopulating HSCs is decreased (5). However, presence of fetal liver hematopoiesis in $S l$ homozygous mutant mice, and persistence of HSC, albeit at reduced levels, in $S l / S l^{d}$ and viable $K L$ mutant mice suggests that $\mathrm{KL}$ is not the sole cytokine responsible for HSC proliferation and maintenance.

The second cytokine cloned with effects on HSC is the ligand for fetal liver tyrosine kinase-3 (FL). As with $\mathrm{KL}$, FL affects proliferation and differentiation of primitive progenitors in vitro. Animals with a homozygous deletion of the genes for either FL or its receptor (Flt3-R) are viable, and only the B-lymphoid lineage appears affected $(6,7)$ by the low population of HSCs. Crossing the Flt3R mutation into the $\mathrm{Sl} / \mathrm{Sl}^{\mathrm{d}}$ genetic background yields mice with a severe decrease in blood counts and HSCs, leading to early death from hematopoietic failure. Thus, KL and FL appear to act in a synergistic manner

\section{Figure 1}

Several factors appear to play a role in the engraftment of hematopoietic stem cells (HSCs) in the bone marrow (BM). HSC (blue nuclei and cytoplasm) must gain access to the bone marrow through the vasculature (red) in a process termed homing, take up residence in the $\mathrm{BM}$, and then undergo self-renewing cell divisions to produce a larger pool of HSC, and differentiate to generate more committed progenitors (other cell types indicated). Although cytokines and growth factors that play a role in HSC differentiation are well known, less is known of cytokines and signals that support HSC expansion. Along with the better studied c-Kit-ligand (KL) and, possibly, the ligand for the receptor Flt3 (FL), thrombopoietin (TPO) is now proposed to play an important role in HSC expansion in the engraftment process.

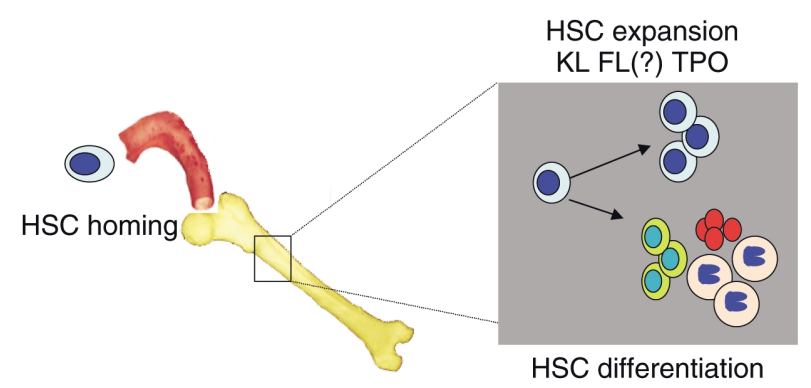


on HSC proliferation and maintenance (6). However, the existence of residual hematopoiesis in these animals indicates that other factors must support HSC proliferation and differentiation.

One such factor may be thrombopoietin (TPO). TPO, the ligand for $\mathrm{Mpl}$, was cloned now almost a decade ago as the long-elusive cytokine responsible for the regulation of megakaryocyte proliferation and differentiation (8). In vitro, TPO increases megakaryocyte proliferation and differentiation (8). Animals in which TPO or Mpl is deleted are viable, but have significantly decreased platelet as well as megakaryocyte levels (9). Surprisingly, other committed progenitors that do not express $\mathrm{Mpl}$ are also decreased as a result of significant loss of HSCs (9). Consistent with the notion that TPO affects HSCs is the finding that HSCs are almost exclusively $\mathrm{Mpl}^{+}$, and that TPO induces proliferation of primitive progenitors in vitro and has therefore become an important factor in ex vivo HSC expansion schemes (10). Mimicking what is seen in knock-out animals, congenital amegakaryocytosis - a human disease arising from mutation of the $\mathrm{Mpl}$ receptor and characterized by a significant decrease in platelets and megakaryocytes - is also associated with the development of aplastic anemia (11). Thus, along with KL and FL, TPO is important for HSC maintenance or expansion in vivo. Whether TPO is responsible for the remaining hematopoiesis in animals in which both Flt3-R and c-Kit are mutated has not yet been tested.

In this issue of the JCI, Fox et al. tested the role of TPO in HSC engraftment (12). They found that 4-fold more BM cells were needed to reestablish hematopoiesis in $\mathrm{Tpo}^{-/-}$than in wild-type mice. Moreover, HSCs expanded 20 -fold less in $\mathrm{Tpo}^{-/-}$mice than in wild-type controls, when evaluated by transplanting BM from primary $\mathrm{Tpo}^{-/}$or wild-type recipients into secondary recipients. Significant- ly, this loss of HSC expansion in vivo could be substantially reversed when $\mathrm{TpO}^{-/-}$mice were supplemented with physiologically relevant amounts of TPO. Thus, like SCF (5), TPO is one of the cytokines responsible for in vivo HSC expansion, possibly by enhancing both HSC proliferation (10) or HSC survival (13).

The only cytokine commonly used to date to hasten neutrophil recovery following transplantation is granulocyte-colony stimulating factor (G-CSF). However, G-CSF does not affect HSC proliferation and therefore does not influence long-term hematopoietic reconstitution. A similar scenario might now be envisaged for TPO: Administration of TPO may enhance both short-term and long-term hematopoietic engraftment in patients who receive limiting numbers of HSCs, such as in adult recipients of cord blood grafts, or in patients who undergo autologous transplantation. However, this question still needs to be addressed directly, as transplants done in the present study did not use limiting numbers of HSC. In addition, since the experiments reported here employed animals with no circulating TPO - unlike thrombocytopenic patients, who usually have high levels of circulating TPO (14) - the effects of TPO supplementation on HSC expansion are difficult to extrapolate from the mouse study. However, systemic administration of G-CSF affects neutrophil recovery despite the high levels of G-CSF levels in myeloablated patients (15). By analogy, it is possible that TPO administration even in patients with high TPO levels will enhance HSC proliferation or survival and lead to higher levels of HSC engraftment. Finally, because of immune mediated complications of TPO administration, TPO is currently not used therapeutically (16). If further studies confirm that TPO can allow transplantation of sub-therapeutic doses of HSC in lethally irradiated wild-type recipients, a clinically suitable source of TPO will need to be identified for this therapy to become practically applicable.

1. Glimm, H., et al. 2001. Previously undetected human hematopoietic cell populations with short-term repopulating activity selectively engraft NOD/SCID-beta2 microglobulin-null mice. J. Clin. Invest. 107:199-206.

2. Ogawa, M., Hanada, K., Hamada, H., and Nakauchi, H. 1996. Long-term lymphohe matopoietic reconstitution by a single 34-low/negative hematopoietic stem cell. Science. 273:242-245.

3. Bernstein, A., Forrester, L., Reith, A.D., Dubreuil, P., and Rottapel, R. 1991. The murine W/c-kit and Steel loci and the control of hematopoiesis. Semin. Hematol. 28:138-166.

4. Zsebo, K.M., et al. 1990. Stem cell factor is encoded at the Sl locus of the mouse and is the ligand for the c-kit tyrosine kinase receptor. Cell. 63:213-224

5. Miller, C.L., et al. 1996. Studies of W mutant mice provide evidence for alternate mechanisms capable of activating hematopoietic stem cells. Exp. Hematol. 24:185-196.

6. Mackarehtschian, K., et al. 1995. Targeted disruption of the $\mathrm{flk} 2 / \mathrm{flt} 3$ gene leads to deficiencies in primitive hematopoietic progenitors. Immunity. 3:147-157.

7. McKenna, H.J., et al. 2000. Mice lacking flt3 ligand have deficient hematopoiesis affecting hematopoietic progenitor cells, dendritic cells, and natural killer cells. Blood. 95:3489-3407.

8. Kaushansky, K., et al. 1994. Promotion of megakaryocyte progenitor expansion and differentiation by the c-Mpl ligand thrombopoietin. Nature. 369:568-571.

9. Alexander, W.S. 1999. Thrombopoietin and the $\mathrm{c}-\mathrm{Mpl}$ receptor: insights from gene targeting. Int J. Biochem. Cell. Biol. 31:1027-1035.

10. Borge, O., Ramsfjell, V., Cui, L., and Jacobsen, S 1997. Ability of early acting cytokines to directly promote survival and suppress apoptosis of human primitive $\mathrm{CD} 34+\mathrm{CD} 38$ - bone marrow cells with multilineage potential at the single-cell level: key role of thrombopoietin. Blood. 90:2282-2292.

11. Ballmaier, M., et al. 2001. c-mpl mutations are the cause of congenital amegakaryocytic thrombocytopenia. Blood. 97:139-146.

12. Fox, N., Priestley, G., Papayannopoulou, T. and Kaushansky, K. 2002. Thrombopoietin expands hematopoietic stem cells after transplantation. J. Clin. Invest. 110:389-394. doi:10.1172/JCI200215430.

13. Pestina, T.I., Cleveland, J.L., Yang, C., Zambetti, G.P., and Jackson C.W. 2001. Mpl ligand prevents lethal myelosuppression by inhibiting p53dependent apoptosis. Blood. 98:2084-2090.

14. Fielder, P.J., et al. 1996. Regulation of thrombopoietin levels by c-mpl-mediated binding to platelets. Blood. 87:2154-2162.

15. Schriber, J.R., et al. 1994. Granulocyte colonystimulating factor after allogeneic bone marrow transplantation. Blood. 84:1680-1684.

16. Basser, R.L., et al. 2002. Development of pancytopenia with neutralizing antibodies to throm bopoietin after multicycle chemotherapy supported by megakaryocyte growth and development factor. Blood. 99:2599-2602. 